\title{
Exploring the potential for secondary uses of Dementia Care Mapping (DCM) data for improving the quality of dementia care
}

\author{
Shehla Khalid \\ (Faculty of Health Studies,) University of Bradford, UK \\ Neil Small \\ Dan Neagu \\ Claire Surr
}

\begin{abstract}
The reuse of existing datasets to identify mechanisms for improving healthcare quality has been widely encouraged. There has been limited application within dementia care. Dementia Care Mapping (DCM) is an observational tool in widespread use, predominantly to assess and improve quality of care in single organisations. DCM data has the potential to be used for secondary purposes to improve quality of care. However, its suitability for such use requires careful evaluation. This study conducted in-depth interviews with 29 DCM users to identify issues, concerns and challenges regarding the secondary use of DCM data. Data was analysed using modified Grounded Theory. Major themes identified included the need to collect complimentary contextual data in addition to DCM data, to reassure users regarding ethical issues associated with storage and reuse of care related data and the need to assess and specify data quality for any data that might be available for secondary analysis.
\end{abstract}

Keywords

Dementia Care Mapping, DCM, secondary use of data, quality of care, data quality, grounded theory

\section{Introduction}

With the aging population bringing about an increasing prevalence of dementia and associated economic, social, health and personal care costs, dementia has been made an international health priority (Knapp et al., 2007; World Health Organization and Alzheimer's Disease International, 2012; Prince et al., 2015). While there is extensive research into cure and symptom amelioration, improving the quality of life and quality of care for people living with the condition is a major global concern (WHO, 2012). Governments, health and socialcare providers, dementia-care organisations and charities therefore, promote not just medically driven research and interventions for the treatment and cure of dementia, but also social and psychological support and research for improving the quality of life and the quality of care.

In 2013, a global dementia summit brought many countries together to pledge to improve dementia care (Department of Health, 2013). One major commitment within the resulting 
action plan was to commence initiatives for integrating data to enhance the opportunities for dementia research (Department of Health, 2013). In response to this, a recent report published by the Organisation for Economic Co-operation and Development (OECD) (Deetjen et al., 2015) proposed a big-data solution for dementia research. It defines big data as both medically driven and non-medically driven data for dementia research, with the term 'nonmedically driven data' referring to data about people's lifestyles, diets and food choices. The OECD report asserts that secondary analysis or reanalysis of currently available data across these medical and non-medical dimensions collectively, could support improvements to the care of people with dementia (Deetjen et al., 2015). While the current focus of the big-data proposal is to enhance research to support better dementia diagnosis, treatments, and a cure, dementia care related data could also provide a resource for understanding, assessing, comparing and improving existing care provision for people with dementia. However, to make dementia care related data part of 'big-data', appropriate datasets must be identified and examined for their suitability for potential secondary uses.

Dementia Care Mapping (DCM) (Bradford Dementia Group, 1997;2005), an internationally used dementia care quality improvement tool (Brooker \& Surr, 2005;2010), offers a dataset with considerable potential to improve quality of care beyond the settings in which it is collected and to enhance dementia care research.

\section{Dementia Care Mapping (DCM)}

DCM is an observational tool set within a practice development process for assessing and improving dementia care quality within formal dementia care settings (hospitals, care homes, day centers) (Bradford Dementia Group, 2005). It involves continual observation of between 1 and 8 people with dementia over a period of time, usually between two and six consecutive hours, by a trained individual called a "mapper" (Bradford Dementia Group, 2005). During a single period of observation (called mapping), large amounts of data are collected. This can offer a rich picture of the person's care experience, including the overall quality of care and factors that contribute to it. A full description of DCM can be found in Brooker and Surr (2006). However, in summary, the mapper records pre-defined codes representing each person's behaviours (Behaviour Category Codes; BCC), mood and engagement (Mood and Engagement Codes; ME) and the quality of staff interactions with the person with dementia (Personal Enhancers; PE's and Personal Detractions; PDs). Alongside this structured coding, qualitative data, in the form of notes about observations are also collected, providing additional information about the structured codes and the physical and social care environment. Once the observations are complete, a mapper processes the raw data and produces individual and group level summaries, such as percentages of time spent in each BCC and ME, the total number of PDs and PEs, and the average ME value over the observation period. This provides an indicator of the relative level of well or ill-being experienced during the mapping period (known as the well or ill-being - the WIB score) (Brooker \& Surr 2010). More detailed care quality indices can be completed by combining information about behaviour, mood and engagement to produce, for example, indicators of agitation, withdrawal and engagement in activities (Brooker \& Surr, 2005).

DCM has been used internationally as both a research tool and, more frequently, in practice development (Brooker, 2005: Younger \& Martin, 2000: Cooke \& Chaudhury, 2012). For practice development, DCM is conducted in a cycle of five phases including; briefing staff within the setting to be mapped about what DCM will involve, conducting the mapping observations, analysing the data and producing a report on the findings, feeding back the 
findings to staff and making action plans for improving care. The data and subsequent action plans can support care improvements to be made at individual patient, care setting (e.g. a hospital ward, day care center, and care home) and organisational (a body with one or more individual care settings) levels (BSI, 2010). For example, at a care setting level, DCM data has been used by managers for overall care quality monitoring (Packer et al., 1997) and identification of staff training and development needs (Lintern et al., 2002). Further, DCM data taken from many care settings within one organisation can be used to audit levels of care and may be combined to identify general areas of good practice across an organisation (Baker, 2014). When used for practice development purposes, DCM is usually the sole data collected and is characteristically in a paper-based format, supported by analysis using basic spreadsheets and reporting using word processing software.

Within a research context, DCM has been used as an outcome measure for assessing behavioural patterns, levels of wellbeing, the quality of interactions with staff and quality of life of people with dementia (Brooker, 2005). It has been used to assess the efficacy of staff training, care quality and culture change projects (Lintern et al., 2002) as well as the efficacy of a range of interventions, including aromatherapy (Baker 2014), intergenerational activity programs (Jarrott \& Bruno, 2003), horticultural therapy (Gigliotti \& Jarrott, 2004) and reminiscence programs (Brooker \& Duce, 2000). Where DCM is used as a research outcome measure, the cyclic practice development process is not usually followed and just the mapping and data analysis stages are completed. Standard statistical analysis software is commonly used for analysis, permitting superior manipulation of the data with more complexity than can be achieved from spreadsheets.

In both practice development and research contexts use of DCM data primarily remains at a local level. That is, the individual (mapper or organisation or research study) collecting the data uses it within that setting or research project for the purpose it was originally collected (primary use). Despite the widespread primary uses of DCM and its importance for improving the quality of dementia care there has, to date, been a limited effort to explore potential for bringing together these multiple datasets and for reanalysis of that data (secondary use). In order to assess the potential of DCM data for secondary analysis, a clear understanding of the technical issues associated with combining these datasets in a repository known as a data warehouse and the social issues associated with mapper and end users issues, concerns and requirements for such a data warehouse is needed. Data warehousing involves collecting and combining data from multiple sources into a data repository which stores data in a specific format (Inmon, 2012). While a previous study (Khalid et al., 2010) proposed a data warehouse as a technical solution for managing DCM data for potential secondary uses, without careful consideration of both technical and social issues, any future developments may encounter user acceptability problems which would impact on success of any technical solution for DCM.

Therefore, this study aimed to investigate mappers' perspectives on the potential for, issues with and concerns about secondary uses of DCM data in the context of future development of a data warehouse.

\section{Methods}

\section{Design}

No research has previously been conducted regarding end users' perspective on the potential secondary use of DCM data. Thus a qualitative methodology incorporating modified 
grounded theory (Strauss \& Corbin, 1998), designed to understand study participants' views at a conceptual level, was employed.

\section{Ethical issues}

Approval from the Humanities, Social and Health Sciences Research Ethics Panel at the University of Bradford, was obtained.

\section{Participants}

A purposive sample of 29 DCM mappers representing three main categories, researchers, trainers and practitioners was identified. These categories reflected the different roles people trained to use DCM might adopt. Researchers are those who use DCM for research purposes, trainers are experienced DCM practitioners who are trained and licensed to deliver DCM training to others and practitioners are those who use DCM for practice development purposes in care provider organisations. There was some overlap between the categories, since DCM trainers might also be using the tool for practice development and/or for research. There were two main reasons for approaching trained mappers as participants in this study. Firstly, they have a good understanding of DCM data due to their training in and experience of using DCM. Therefore, they are well placed to understand issues and challenges associated with secondary analysis of DCM data. Secondly, mappers' views have always been considered important for any new development in DCM (Innes \& Surr, 2003; Brooker \& Roger, 2001; Brooker \& Surr, 2006) and are integral to acceptance of any development.

\section{Procedure}

Recruitment. Potential participants from a range of UK-based organisations involved in dementia-care provision, monitoring, regulation and research, were contacted to take part in the study. They were identified through existing networks of the authors and via reviewing authorship of relevant published research. DCM users were also contacted via an existing database of trained mappers held by the School of Dementia Studies at the University of Bradford. Potential participants were sent personalised emails inviting them to take part in the study and asked to reply if they were interested. One further reminder was sent to those who did not respond within two weeks. Those who replied were contacted again to arrange a time for interview at a location (face to face, or via Skype or phone), date and time convenient to them.

Consent. Written informed consent was obtained from all participants ahead of interviews commencing.

Interviews. To encourage the exploratory process, a semi-structured interview guide with broad topics was developed, which helped the study participants to talk about their perceptions, views, issues and concerns regarding the potential secondary uses of DCM data. Nineteen interviews were conducted face-to face and six were via Skype, all were audio recorded and transcribed. Interviews ranged in length from 45 to 60 minutes. Each participant was interviewed only once.

A total of 29 interviews were conducted over three phases. See table 1 for participant details. During the first phase, 10 interviews were conducted with mappers spanning a variety of roles (practitioners, trainers and researchers) to explore their current use and their views about potential uses of DCM data. The second phase of data collection consisted of semistructured interviews with 14 more participants (e.g. DCM trainers, researchers and 
practitioners) in order to collect more focused and detailed data. During the third phase interviews were conducted with five researchers to further explain and clarify emerging views and issues.

\section{Data analysis}

Transcribed data from the interviews was managed using QSR Nvivo 9 (Bringer et al., 2006) and analysed using Strauss and Corbin's (1998) open and axial coding techniques. Open coding is the initial step of data analysis using grounded theory, which Strauss and Corbin (1998) refer to as examining data at a micro level (i.e., looking at small details). The open coding identified a number of descriptive codes. These codes were merged into categories, based on conceptual similarities, where various aspects of the data were compared to formulate the differences and similarities within them. The emerged categories were linked based on the conceptual relationships between the data and high-level categories were formulated (axial coding) (Strauss \& Corbin, 1998). Both open and axial coding continued until data was saturated and no new concepts and their relationships were emerging from the subsequent interview data. All coding was undertaken by (SK) and reviewed by (CS and NS) to check for agreement/accuracy of themes.

\section{Findings}

Three categories emerged: Potential secondary uses of DCM data; additional data requirements; and quality and security of DCM data for secondary uses.

\section{Potential secondary uses of DCM data}

The interview data highlighted that potential secondary uses of DCM data varied according to the previous experience people had. DCM Practitioners who were using DCM for practice development purposes largely within their own organisations, envisaged secondary analysis of DCM data could provide a mechanism for sharing good practice through processes such as benchmarking. DCM trainers, on the other hand, were interested in having access to historic and integrated DCM data to see trends and patterns, to identify and share good care practices and to use a variety of examples from the data within their DCM teaching. DCM researchers saw benefits in using DCM data to contribute to future research for example as a dataset they could draw on to conduct secondary research.

Benchmarking. Benchmarking, defined as 'the continuous and collaborative process of measuring and comparing the outcomes of key work processes with those of the best performers in evaluating organisational performance' (Lovaglio, 2012, p. 2), is a method organisations may use in order to make continuous quality improvements (Ettorchi-Tardy et al., 2012). Study participants saw this as a concept to identify a mechanism by which secondary DCM data could potentially be used to improve care quality. They saw two mechanisms for achieving this. The first was through using DCM data for internal benchmarking; meaning comparing either best practice with current practice within their own organisation (Lovaglio, 2012). This would allow an examination of how far the quality of care they deliver was consistent across all parts of the organisation.

\footnotetext{
It would be lovely if they [mappers] mapped in surgery and we could compare, yes, because it is a totally different environment and way of working, but if we are working really good, then it would be nice to see if we could transfer it or use it across. And for different areas....I mean. It
} 
would be interesting seeing what they do and what could we do.' (Practitioner, 12)

Another potential use of DCM data was for external benchmarking. Here study participants expressed a desire to measure their own organisation's performance against that of other similar organisations.

'....although our service is quite unique, but knowing about other services within the country and further afield, I think, would be really interesting.' (Practitioner 2)

One participant saw this as a way of validating their own organisation's standing, it would allow them to show that;

'We have reached that level of care, we are providing person-centred care and we can prove this through DCM' (Practitioner Trainer 10).

This indicated that some mappers hoped that benchmarking might be able to offer a quantitative measure for what 'good quality care' or 'person-centred care' looks like and against which organisations could appraise their own performance.

DCM teaching, training and support. The DCM trainers saw value in using DCM data for secondary purposes to improve training provision and to track trained mappers' professional development over time.

I think it would be interesting to know the extent to which people [mappers] go on to actually use the tool after the training or particular reasons why they do and do they need support in using them? If they are not using it, then why they haven't managed to use it. I think that would be interesting to know.' (Practitioner Trainer, 24)

In addition to having access to data on the use of DCM by individual mappers, most DCM trainer interviewees also agreed that secondary data could enable them to learn from others' use of DCM and to identify examples of good care practices that could be used during delivery of DCM training courses.

I think one of the things that across care settings says that we are not sharing good practice. There is a lot we can do in sharing to trigger ideas and factors.' (Trainer, 18)

'One of the things is that, during the course, you can enrich the evidence with examples, which is possible by having access to the various types of data from this data warehouse.' (Trainer, 26)

Therefore, for trainers, a DCM data warehouse could enable information on DCM use to be made available, which could strengthen the ways in which they could better enable the use of DCM in practice through the training course and ongoing mapper support.

Secondary research. DCM researchers welcomed the potential use of DCM data for secondary research purposes. They hoped access to a data warehouse would permit exploration of data collected from a variety of care settings, across a range of geographical locations, over time, and for a multiplicity of purposes for the purpose of secondary analysis. 
'I would be very fascinated in being able to extract back the information [DCM data] to see what behaviours people [residents] are experiencing in particular... if there is another organisation that is looking at mapping in a continuing care setting and being able to extract that for comparing and contrasting. So that is linking different organisations and settings together.' (Researcher Practitioner Trainer, 17)

Researchers also saw a DCM data warehouse as offering potential cost and time savings for researchers.

'I would love to use that data [secondary DCM data]. Because, now, so much time is taken doing mapping ourselves, which is enormous in time investment. It would be a great opportunity if you had a sort of a database where everyone put their data in and you can also use it for research purposes.' (Researcher, 13)

'It would be extremely helpful, because now I have only my little sample to study my research question. But it would be really interesting, as it has not been studied before and it's a limitation of my study that there is such a small sample. So I would be really interested in using other mappers' data as well to have a bigger sample to study my research question.' (Researcher, 4)

While researchers saw wide benefits to the potential development of a DCM data warehouse, they were also clear in identifying a range of conditions that would need to be met in order for the data in the warehouse to be useable for secondary purposes. These included the need for additional data and for reassurance about data quality.

\section{Additional data requirements}

Interviewees identified that in order to be useful DCM data would need to be accompanied by a range of additional data within a warehouse, before they could use the data for research.

'I think it depends what other information is available on the study databases. If it was just DCM data with nothing else, then I think from... As a part of the dementia organisation, it would be valuable for us but as a researcher it would not be seen as particularly useful. But if you have neuropsychiatric inventory data, information on cognitive data, functional data, alongside, I think that would allow you to ask some interesting questions.' (Researcher, 20)

Additional data identified as required to provide crucial context to the DCM data could concern the individuals with dementia who had been mapped, the care setting where the observations were undertaken and details about staff members on duty during the mapping. The researchers were clear that without this data, it was unlikely that they would find DCM data useful for secondary research.

'I think you should think about this really hard upfront. Otherwise, the data [DCM data] cannot be used, if you don't have all the required information. Like use of medication and all that information should have been in the database.' (Researcher, 13) 


\section{Quality and security of DCM data for secondary uses}

Mappers across all three interviewee types raised concerns regarding the quality and security of DCM data in the context of its secondary use. This included the reliability and validity of the DCM data included in the warehouse, Reliability of DCM data is assessed through the inter-rater reliability (IRR) process, which is where two or more mappers conduct mapping on the same people with dementia at the same time and compare the similarities and differences within their coded data (Bradford Dementia Group 2005). They can work out a percentage of concordance which needs to be over $70 \%$ before data can be used for practice development purposes and $80 \%$ for research. For study participants, this inter-rater reliability of data was considered a significant factor in determining the quality of DCM data for secondary uses.

\section{'You make reference to other people's [DCM] data but you cannot be sure of the reliability of it. You would never be able to be certain of the reliability of other people's data.' (Researcher Practitioner Trainer, 16)}

As any secondary user of the data would not be the person or organisation involved in collecting the data they need to be reassured, via the data warehouse, that the data is of acceptable quality. Interviewees identified that this might be achieved by including information in the warehouse on mappers' training level, experience and IRR score(s) alongside their DCM data.

'I want to see how the mappers have scored, if they are two mappers, have they correlated with each other to see if it [score] matches.' (Researcher, 13)

Researcher 4: 'She [friend] is a DCM trainer but with gold standard. But I am worried that, if I am going to use data from the regular mappers, how the quality of data I am going to look at...'

SK: What do you mean by a trainer with "gold standard"?

Researcher 4: 'I mean she has a lot of experience of mapping and she has trained many mappers so far and she is lead of DCM here in this country.'

It was only through providing this level of detail that the data in the warehouse would be seen as trustworthy by the potential end users. DCM researchers also raised concerns regarding ethical considerations that must to be adhered to when using healthcare data for research purposes. They stipulated a requirement to be able to find, alongside the DCM data, information including any approvals, consent processes and allied documentation.

\section{'It's very important that data is collected considering all ethical aspects. As a researcher, I would like to see what ethical considerations were taken when mapping was done.' (Researcher, 25)}

While consent for observation is always gained when using DCM the information given, reasons for data use and informed consent procedures are not as rigorous in practice development as they are for research, as was highlighted by one respondent. 
'So, for example, you imagine if it was your service and you know you were a key worker for five or six people and you measure their care using DCM, then I suppose, then you might not get ethical approval and you might not even get consent. You just do it as part of the care planning and I think there are issues around then what you can do with your data. The data stays as part of your care plan within your service and, if it is not shown to anybody else, then it's fine. But when you start sharing all that anonymously, I think there will be ethical considerations around that, which need careful thought.' (Researcher, 25)

The interviewees also highlighted that, in addition to usual ethical processes there may be additional information for participants and formalised within consent forms to allow the retention and sharing of anonymised data for secondary use.

'The only thing, which is important, is that, if you collect data, you are doing it for one purpose and, if you use that data for other purposes, then you have to inform the care organisations where you mapped that you are going to share the data with other researchers. Because that is not the thing that you tell them at the start. So I think it is very important.' (Researcher, 4)

\section{Discussion}

\section{DCM data for benchmarking}

A successful benchmarking process requires data which is suitable, available, complete and accurate and that is comparable across settings (Campbell et al., 2003: Nolte, 2010). While our data reveals that those practitioners who use DCM data for internal benchmarking see additional value for external benchmarking, the feasibility of using DCM data in this way needs further exploration.

DCM data is suitable in terms of providing a WIB score as an indicator to assess changes in care over time (Brooker et al., 1998). While there are suggestions in the literature that the mean WIB score across similar types of care settings can be used to indicate differences in care across setting types, there is no further evidence of the applicability of this data for identifying best practice or benchmarking. DCM data has also been used for comparison purposes while assessing changes in care across a number of settings (Brooker et al., 1998; Kuhn et al., 2002).

Based on our findings and the literature review, the use of DCM data seems promising both in terms of users' acceptability and the suitability of DCM data for benchmarking.

\section{DCM data for secondary research}

Reanalysis of DCM data for secondary purposes is infrequently reported in the literature. Examples of where it is used within the context of secondary research come from Fossey, Lee and Ballard (2002) who conducted secondary analysis of DCM data from three earlier studies in order to examine the psychometric properties of DCM as a research outcome measure and Innes and Surr (2001) who undertook a cohort analysis of data from two studies to describe standards of dementia care in UK care homes. Our findings show that study participants see 
value in reusing DCM data for secondary research. However, our study also underlines the need for additional information alongside the DCM data to make it a richer source for secondary research purposes. In reporting this we are consistent with other research that has discussed the need to report: participant characteristics such as age, gender, ethnicity, length of stay in health and social care facilities (Kuhn et al., 2005: Barnes et al., 2013); depression levels (Kuhn et al., 2004) and cognitive state (Kuhn et al., 2002); care setting characteristics such as type, location and size (Willemse et al., 2011) and staff-related information such as staff ratios and training (Gigliotti et al., 2004). This additional data is not routinely collected in a standard mapping process although it may be available from other sources such as electronic patient records.

Within a secondary analysis, the DCM data and additional information about care settings, patients and staff could be explored to investigate potential associations that may only be identified through combining data from a large number of maps across a wide range of settings. The integration of such information could facilitate designing specific tailored interventions for similar patient groups to improve their care. Further, DCM data, alongside some additional information, could provide an opportunity to perform in-depth analysis about what, where, why and how well-being of people with dementia is influenced in formal care settings.

\section{Quality and security of DCM data for secondary uses}

Mappers expressed concerns regarding the quality and security of DCM data for secondary uses, particularly for secondary research. DCM data quality is an under-researched area. While for the primary use of data the literature highlights that data quality is associated with the mapper's IRR score (Brooker et al., 1998; Thornton et al., 2004), there is a lack of any criteria for the quality of DCM data for secondary uses. According to the study participants, the mapper's reliability, their experience and training levels, are significant for the secondary use of DCM data, particularly for research purposes. Further research is required to identify how mappers' reliability for producing good quality DCM data can be regulated and monitored.

Further, our study corroborates what other studies have reported in terms of ethical requirements for healthcare data. While primary use of healthcare data is concerned with patients' own care and treatment, where practitioners or care providers are obliged to protect patients' privacy, secondary use of the same data, particularly for research purposes, can raise additional data-protection and privacy concerns (Wiesenauer et al., 2012; Lamas et al., 2015). While it is necessary to gain patients' consent for the secondary use of their data, the secondary use of DCM data might face additional consent-related issues. This requires establishing procedures that ensure an effective process of consent for the potential future secondary uses of DCM data.

\section{Limitations}

This study is limited to exploring only the views and perceptions of those individuals who were recruited based on their knowledge of DCM as mappers. It is important to seek views from those who might not be mappers but who would like to use DCM data for quality improvement and research purposes. These could be care-quality improvement organisations, universities and dementia charities.

\section{Conclusions}


The potential to utilise data sharing has attracted considerable interest in health and social care. This interest has been made possible by the increasing ubiquity of digitalisation and by enhanced technical possibilities in manipulating and sharing large and complex data sets. Even in the recent past, for example in the years when DCM was first used, these possibilities were either not there or the possibilities inherent in digital data were not so evident. But data sharing is not just a technical solution waiting to discover a research question. It is also attracting interest because of the cost benefits of reusing often expensively collected data. There are also ethical considerations that underline the need to maximise the usefulness of the data we get from people who are often sick and under considerable pressure, characteristically this is hard gained and generously given personal information that we need to make full use of. There are also academic reasons inherent in a recognition that we can do some things with large data sets, collected in multiple settings, that cannot be done with single site/single organisation studies. Many studies concerned with care of older people in general and of people with dementia in particular have been small scale studies. While these can be very valuable, utilising big data offers the possibility of a step change in the assessment of the efficacy of interventions and in the comparison of care modalities. DCM data has been collected, over twenty years, in a wide range of settings in different countries. It is rich and complex data that has used standard tools and been collected by people trained using the same protocols, and it has been stored in similar ways. It is data that relates to an identifiable population group in care settings that include considerable similarities. As such this is data that appears very well suited for secondary uses. However to be able to ensure its potential secondary uses it must be stored in ways that permit secondary use and it must be made available in ways that facilitate reliable access, made as easy as possible.

This paper has argued that DCM has potential to provide a basis for a dementia care quality dataset that may be used for secondary analysis purposes within practice and research contexts. It has also emphasised that the potential secondary uses of DCM data require the users to know that data they are using has been properly collected, meeting ethical standards and standards of accuracy and completeness. Extra categories need to be built into DCM data collection to routinely provide this and links made with other data sets that would allow for access to a more complete picture of the individual being mapped. To achieve this broadening of the scope and deepening the detail encompassed by DCM changes in training are required. The potential secondary use of DCM data will consequently enhance the national and international data-sharing culture in the DCM community and opportunities to make DCM data part of big-data for dementia research.

\section{Declaration of conflicting interests}

The author(s) declare(s) that there is no conflict of interest. 


\section{References}

Baker, C. (2014) Developing excellent care for people living with dementia in care homes. London: Jessica Kingsley

Ballard, C., Powell, I., James, I., Reichelt, K., Myint, P., Potkins, D., ...Barber, R. (2002) Can psychiatric liaison reduce neuroleptic use and reduce health service utilization for dementia patients residing in care facilities. International Journal of Geriatric Psychiatry, 17(2):140-145.

Barnes, S., Wasielewska, A., Raiswell, C., \& Drummond, B. (2013). Exploring the mealtime experience in residential care settings for older people: an observational study. Health and Social Care in the Community, 4(21):442-450.

Bradford Dementia Group (1997) Evaluating dementia care: the DCM method 7th edition. University of Bradford, Bradford, England, UK.

Bradford Dementia Group (2005). DCM 8 User's Manual. University of Bradford, Bradford, UK.

Bringer, D.J., Johnston, H.L., \& Brackenridge, H.C., (2006) Using computer-assisted qualitative data analysis software (CAQDAS) to develop a grounded theory project. Field Methods 18, 245-266.

Brooker, D. J., Foster, N., Banner, A., Payne, M., \& Jackson, L. (1998) The efficacy of Dementia Care Mapping as an audit tool: report of a 3-year British NHS evaluation. Aging \& Mental Health 2 (1), 60-70.

Brooker, D. J., (2005). Dementia care mapping: a review of the research literature. The Gerontologist. 45 Spec No 1(1):11-8.

Brooker, D. J., \& Duce, L. (2000) Wellbeing and activity in dementia: a comparison of group reminiscence therapy, structured goal-directed group activity and unstructured time. Aging \& Mental Health. 4(4): 354-358.

Brooker, D. J., \& Rogers, L. (2001) Dementia Care Mapping (DCM) think tank transcripts,. Bradford University, England UK, Bradford University.

Brooker, D. J., \& Surr, C. (2005) Dementia care mapping: principles and practice. University of Bradford: University of Bradford, UK.

Brooker, D. J., \& Surr, C. (2006) Dementia Care Mapping (DCM): initial validation of DCM 8 in UK field trials. International Journal Of Geriatric Psychiatry 21 (11), 1018-1025.

Brooker, D. J., \& Surr, C. (2010) Dementia care mapping : principles and practice. 3rd ed. Bradford: University of Bradford, UK.

BSI (2010) Use of Dementia Care Mapping for inproved person-centred care in a care provider organisation. London: BSI. 
Campbell, S. M., Braspenning, J., Hutchinson, A., \& Marshall, M. N. (2003) Research methods used in developing and applying quality indicators in primary care. British Medical Journal 326, 816-819.

Cooke, H.A., \& Chaudhury, H. (2012) An examination of the psychometric properties and efficacy of Dementia Care Mapping. Dementia.

Department of Health (2013) G8 Dementia summit declaration. London, UK: Department of Health. Retrieved from https://www.gov.uk/government/publications/g8-dementiasummit-agreements/g8-dementia-summit-declaration

Deetjen, U., Meyer, E. T. and Schroeder, R. (2015) Big data for advancing dementia research: an evaluation of data sharing practices in research on age-related neurodegenrative diseases. Paris, France, OECD Publications.

Ettorchi-Tardy, A., Levif, M., \& Michel, P. (2012) Benchmarking: a method for continuous quality improvement in health. Health Policy 7 (4), 101-119.

Fossey, J., Lee, L., \& Ballard, C. (2002) Dementia care mapping as a research tool for measuring quality of life in care settings: psychometric properties. International Journal of Geriatric. Psychiatry, 17(11): 1064-1070.

Gigliotti, C.M., Jarrott, S.E., \& Yorgason, J. (2004) Harvesting health:Effects of three types of horticulture therapy activities for person's with dementia. Dementia, 3(2): 160-180.

Innes, A., \& Surr, C. (2001) Measuring the well-being of people with dementia living in formal care settings: The use of dementia care mapping. Aging \& Mental Health, $5(3): 258-268$.

Inmon, W.H. (2012) Data warehousing in the healthcare environment, Retrieved from http://inmoncif.com/registration/whitepapers/DATA\%20WAREHOUSING\%20IN\%20H E\%20HEALTHCARE\%20ENVIRONMENTR1.pdf

Jarrott, S.E. \& Bruno, K. (2003) Intergenerational activities involving persons with dementia: an observational assessment. American Journal of Alzheimer's Disease and Other Dementias, 18(1): 31-37.

Khalid. S., Surr, C. \& Neagu, D. (2010) DCM data management framework: A data warehousing approach. International Conference on Information Technology in Bio-And Medical Informatics, Bilbao, Spain: Springer Berlin Heidelberg

Knapp, M., Comas-Herrera, A., Somani, A., \& Banerjee, S. (2007) Dementia: international comparisons, Summary report for the National Audit Office. Personal Social Services Research Unit. Retrieved from http://www.pssru.ac.uk/pdf/dp2418.pdf

Kuhn, D., Edelmann, P., \& Fulton, B.R. (2005) Daytime Sleep and the Threat to Well-being of Persons with Dementia. Dementia. The International Journal of Social Research and Practice, 2(4):233-247. 
Kuhn, D., Fulton, B. R. and Edelmann, P. (2004) Factors influencing participation in activities in dementia care settings. Alzheimer's Care Quarterly 3 (5), 144-152.

Kuhn, D., Kasayka, R. E. and Lechner, C. (2002) Behavioral observations and quality of life among persons with dementia in 10 assisted living facilities. American Journal of Alzheimer's Disease and Other Dementias 17 (5), 291-298.

Lamas, E., Barh, A., Brown, D., \& Jaulent, M. C. (2015) Ethical, legal and social issues related to the health data-warehouses: re-using health data in the research and public health research. Studies in Health technology and Informatics, 2010, 719-23.

Lintern, T., Woods, R.T., \& Phain, L. (2002) Before and after training: A case study of intervention. London: Hawker Publications.

Lovaglio, G.P. (2012) Benchmarking strategies for measuring the quality of healthcare: problems and prospects. The Scientific World Journal, 1-13.

Martin, G.W. \& Younger, D. (2000) Anti oppressive practice: a route to the empowerment of people with dementia through communication and choice. Journal of Psychiatric and Mental Health Nursing, 7(1): 59-67.

Nolte, E. (2010) International benchmarking of healthcare quality: a review of the literature. California, US: RAND Corporation.

Packer, T. \& Jeffries, M. (1997) Halopriedol, Hips and Toenails...... Journal of Dementia, 5(Nov/Dec): 22-4.

Prince, M., Wimo, A., Guerchet, M., Wu, Y. and Prina, M. (2015) The global impact of dementia; an analysis of prevalence, incidence, cost and trends. Alzheimer Disease International.

Strauss, A.L., \& Corbin, J.M. (1998) Basics of qualitative research: techniques and procedures for developing grounded theory $\left(2^{\text {nd }}\right.$ Ed) London, Sage.

Thornton, A., Hatton, C., \& Tatham, A. (2004) Dementia Care Mapping reconsidered: exploring the reliability and validity of the observational tool. International Journal of Geriatric Psychiatry 19 (8), 718-726.

World Health Organisation (2012). Dementia: a public health priority.

World Health Organization and Alzheimer's Disease International (2012) Dementia: a public health priority. Retrieved from http://www.who.int/mental_health/publications/dementia_report_2012/en/index.html

Wiesenauer, M., Johner, C., \& Rohrig, R. (2012) Secondary use of clinical data in healthcare providers - an overview on research, regulatory and ethical requirements. Studies in Health Technology and Informatics 180, 614-618.

Willemse, M.B., Smit, D., De Lange, J., \& Pot, M.A. (2011) Nursing home care with people with dementia and residents' quality of life, quality of care and staff well-being: Design 
of the living arrangements for people with dementia (LAD)-study. BMC Geriatrics, 11(11).

Younger, D. \& Martin, G.W. (2000) Dementia care mapping: an approach to quality audit of services for people with dementia in two health districts. Journal of Advanced Nursing, 32(5): 1206-1212. 
Table 1: Participant details

\begin{tabular}{|c|c|c|}
\hline Participant code & DCM Status & Affiliations \\
\hline Trainer 1 & DCM Trainer & University (UK) \\
\hline Practitioner 2 & $\begin{array}{ll}\text { Basic } & \text { DCM } \\
\text { mapper } & \\
\end{array}$ & Healthcare setting (UK) \\
\hline Practitioner 3 & $\begin{array}{ll}\text { Basic } & \text { DCM } \\
\text { mapper } & \\
\end{array}$ & Healthcare setting (UK) \\
\hline Researcher 4 & $\begin{array}{l}\text { Basic } \quad \text { DCM } \\
\text { mapper }\end{array}$ & Research organisation (non-UK) \\
\hline DCM Practitioner 5 & $\begin{array}{l}\text { Advanced DCM } \\
\text { mapper }\end{array}$ & Healthcare setting (UK) \\
\hline Practitioner 6 & $\begin{array}{ll}\text { Basic } & \text { DCM } \\
\text { mapper } & \\
\end{array}$ & Healthcare setting (UK \\
\hline Practitioner 7 & $\begin{array}{ll}\text { Basic } & \text { DCM } \\
\text { mapper } & \\
\end{array}$ & Healthcare setting (UK) \\
\hline Researcher Practitioner 8 & Advanced mapper & University (UK) \\
\hline Researcher Practitioner 9 & $\begin{array}{ll}\text { Basic } & \text { DCM } \\
\text { mapper } & \\
\end{array}$ & University (UK) \\
\hline Practitioner Trainer 10 & DCM trainer & $\begin{array}{l}\text { Research organisation and local dementia charity } \\
\text { (non-UK) }\end{array}$ \\
\hline Researcher Trainer 11 & DCM trainer & Research organisation (non-UK) \\
\hline Practitioner 12 & $\begin{array}{ll}\text { Basic } & \text { DCM } \\
\text { mapper } & \\
\end{array}$ & Hospital setting (UK) \\
\hline Researcher 13 & DCM mapper & University (non-UK) \\
\hline Practitioner Trainer 14 & DCM mapper & Social care organisation (UK) \\
\hline researcher 15 & Basic mapper & University (UK) \\
\hline Researcher Practitioner Trainer 16 & DCM trainer & NHS trust (UK) \\
\hline Researcher Practitioner Trainer 17 & DCM trainer & NHS trust (UK) \\
\hline Trainer 18 & DCM trainer & University (UK) \\
\hline Trainer 19 & DCM trainer & University (UK) \\
\hline Researcher 20 & Basic mapper & Dementia charity (UK) \\
\hline Practitioner Trainer 21 & DCM trainer & UK \\
\hline Practitioner Trainer 22 & DCM trainer & UK \\
\hline $\begin{array}{l}\text { Practitioner } \\
23\end{array}$ & $\begin{array}{ll}\text { Basic } & \text { DCM } \\
\text { mapper } & \\
\end{array}$ & UK based dementia charity \\
\hline Trainer Practitioner 24 & DCM trainer & University (UK) \\
\hline Researcher 25 & $\begin{array}{ll}\text { Basic } & \text { DCM } \\
\text { mapper } & \\
\end{array}$ & University (non-UK) \\
\hline Practitioner Trainer 26 & DCM trainer & UK \\
\hline Researcher 27 & $\begin{array}{ll}\text { Basic } & \text { DCM } \\
\text { mapper } & \\
\end{array}$ & UK \\
\hline Researcher 28 & $\begin{array}{ll}\text { Basic } & \text { DCM } \\
\text { mapper } & \end{array}$ & Dementia research organisation (Non-UK) \\
\hline Trainer 29 & DCM trainer & Dementia care organisation (Non-UK) \\
\hline
\end{tabular}

